Int. J. Odontostomat.,

4(2):133-137, 2010.

\title{
Resorte de Coffin: Estudio de 2 Diferentes Tipos de Ligas Metálicas
}

\author{
Coffin Appliance: Study of 2 Different Types of Metal Alloys
}

Rogério Lacerda dos Santos \& Matheus Melo Pithon

DOS SANTOS, R. L. \& PITHON, M. M. Resorte de Coffin: Estudio de 2 diferentes tipos de ligas metálicas. Int. J. Odontostomat., 4(2):133-137, 2010.

RESUMEN: La aparición de los alambres con una baja cantidad de níquel son un gran avance en ortodoncia, supliendo la necesidad de ortodoncia para pacientes que pueden tener hipersensibilidad de este ión, pero no se conoce mucho acerca de las propiedades mecánicas de estos alambres. El objetivo de este estudio fue probar la hipótesis de que no hay diferencia en el desempeño mecánico de los alambres de acero inoxidable y alambres de acero inoxidable con bajo contenido de níquel, evaluando la fuerza, resistencia y módulo de elasticidad producida por el resorte de Coffin hecho con alambre de 0,032 y 0,036 pulgadas. Se construyeron 60 unidades de Coffin, 30 para cada tipo de aleación, siendo 15 confeccionadas con alambre de 0,032 pulgadas y 15 con alambre de 0,036 pulgadas. Todos los arcos fueron sometidos a la prueba mecánica de compresión en la máquina EMIC DL-10000, simulando 4, 6, 9 y 12 mm de activación. El análisis de varianza y comparación múltiple (ANOVA) y la prueba de Tukey $(p<0,05)$ fueron utilizados para evaluar la fuerza, resistencia y módulo de elasticidad. Los grupos que utilizaron alambre de 0,036 pulgadas presentaron estadísticamente $(p<0,05)$ mayores niveles de fuerza, resistencia y módulo de elasticidad en comparación con dispositivos con 0,032 pulgadas de alambre para ambas aleaciones. Para el mismo espesor, no hubieron diferencias estadísticamente significativas entre los 2 tipos de aleaciones, con excepción de los dispositivos con activación de $9 \mathrm{~mm}$ y espesor de 0,036 pulgadas que mostraron una diferencia estadística $(p<0,05)$. Los resortes de Coffin evaluados para ambas aleaciones metálicas produjeron fuerzas adecuadas para el tratamiento ortodontico, por lo que debe ser correctamente planificada su aplicación clínica.

PALABRAS CLAVE: mordida cruzada, aparatos ortodóncicos, fuerza, alergia al niquel.

\section{INTRODUCCIÓN}

Los tipos más comunes de aleaciones de acero austenítico utilizadas en ortodoncia, como en el alambre y braquets son del tipo 302 y 304 , según el Instituto Americano del Hierro y el Acero (American Institute of Steel and Iron, AISI), con aproximadamente un $18 \%$ de cromo, $8 \%$ de níquel, que está representado por el grupo de 18-8 de acero inoxidable. El porcentaje de níquel en los alambres de ortodoncia varía desde el $8 \%$ (como el acero inoxidable) a más del $50 \%$ (como en el caso de níquel-titanio) (Eliades \& Athanasiou, 2002; Park \& Shearer, 1983).

El níquel es un fuerte sensibilizador inmunológico y puede producir hipersensibilidad inmune (Janson et al., 1998) y generar reacciones de los tejidos que pueden ser constituidas por zonas difusas de color rojo intraorales, ampollas y úlceras que se extienden a la zona peribucal, además de reacciones urticariforme y eczematosas en la cara o en zonas más distantes del cuerpo (Hensten-Pettersen et al., 2001).

Para resolver este problema, las aleaciones de acero inoxidable con bajo contenido de níquel comenzaron a ser utilizadas en ortodoncia para la confección de diversos tipos de aparatos, entre ellos, los expansores utilizados para la corrección de mordida cruzada dentaria posterior que pueden ser empleados en la dentición temporal, mixta y permanente. Los aparatos comúnmente utilizados para corregir una mordida cruzada dental incluyen al resorte de Coffin, el arco "W" y el arco Quad Hélix (Adams, 1969; Bell \& LeCompte, 1981; Proffit, 1986). El dispositivo de Coffin, 
descrito por Walter Coffin en 1881, fue originalmente utilizado en placas removibles para ampliar arcos estrechos, y su aplicación clínica es con frecuencia recomendada (Adams; Chaconas \& de Alba y Levy, 1977). Teniendo en cuenta esto, el uso de alambre de acero inoxidable con bajo contenido de níquel para la fabricación de estos dispositivos puede ser una alternativa para los pacientes con alto potencial alérgico a los alambres de acero inoxidable convencionales.

El objetivo de este estudio fue probar la hipótesis de que no hay diferencia en el rendimiento mecánico de los alambres de acero inoxidable y alambres de acero inoxidable con bajo nivel de níquel al evaluar la fuerza, resistencia y módulo de elasticidad producida por el resorte de Coffin utilizado para la corrección de la mordida cruzada dentaria posterior y determinar los niveles óptimos de activación para cada aparato.

\section{MATERIAL Y MÉTODO}

Fueron evaluados en este estudio 60 resortes de Coffin, utilizándose dos grosores de alambre (0,032 y 0,036 pulgadas), de 2 tipos de aleaciones, la primera de acero inoxidable (acero inoxidable, $\mathrm{CrNi}$, Morelli, Sorocaba, São Paulo, Brasil) y segundo de acero inoxidable con bajo contenido de níquel, menor al 0,2\% (Biowire-CrMo, Morelli, Sorocaba, São Paulo, Brasil), donde 30 aparatos fueron confeccionados para cada tipo de aleación de alambre, 15 con alambre de 0,032 pulgadas y 15 con alambre de 0,036 pulgadas.

Los resorte de Coffin confeccionados poseían 2 segmentos internos de alambre de $25 \mathrm{~mm}$ de longitud y 1 asa trasera $10 \mathrm{~mm}$ de longitud (Fig. 1).

Las muestras fueron preparadas por el mismo profesional, utilizando un modelo estándar de las distancias intercaninas e intermolares. Un segmento de $5 \mathrm{~mm}$ de longitud de un tubo telescópico de 0,040 pulgadas se adjuntó en el borde posterior de cada extremo externo del resorte de Coffin, a fin de posicionar una aplicación de la fuerza en los primeros molares permanentes. Un segmento de alambre de acero inoxidable de $10 \mathrm{~mm}$ de largo y 0,032 pulgadas de diámetro fue soldado con soldadura de plata en el centro del tubo telescópico de modo que el aparato pudiera ser fijado a la máquina ensayo de universal (Martinelli et al., 2006).

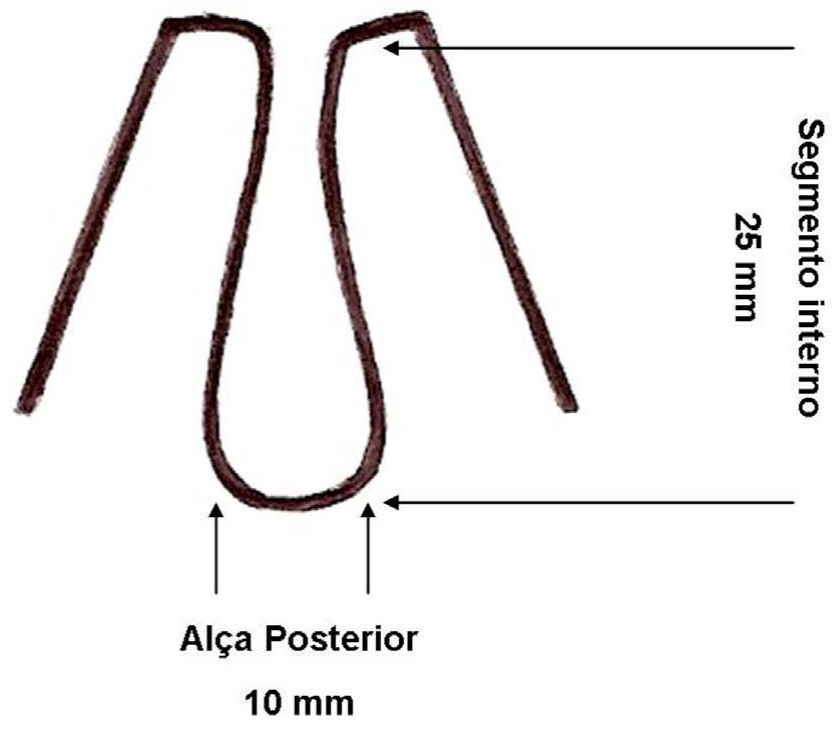

Fig. 1. Ilustración de la figura utilizada para construir el resorte de Coffin.

Cada muestra fue inicialmente activada en 12 $\mathrm{mm}$ y luego sometido a una serie de ensayos de compresión en la máquina EMIC DL 10000, usando el programa Mtest versión 1.0, a una velocidad de 5 $\mathrm{mm} / \mathrm{min}$. El programa Mtest generó la media fuerza, resistencia y módulo de elasticidad producida por la activación de 4, 6, 9 y $12 \mathrm{~mm}$, y el gráfico del comportamiento mecánico en este índice de la activación. Los resultados se compararon estadísticamente mediante un factor ANOVA y el análisis de Tukey.

\section{RESULTADOS}

Fuerza, resistencia y módulo de elasticidad aumentaron proporcionalmente al aumento de las activaciones (Tablas I y II). Además, los grupos que utilizaron alambre de 0,036 pulgadas presentaron estadísticamente $(p<0,05)$ mayores niveles de fuerza, resistencia y módulo de elasticidad en comparación con los aparatos de alambre de 0,032 pulgadas para ambas aleaciones. Hubo una diferencia estadísticamente significativa a las fuerzas generadas entre los grosores de 0,032 y 0,036 pulgadas para todas las activaciones evaluados $(p<0,05)$. Para el mismo grosor de alambre no hubo diferencia estadísticamente significativa entre las aleaciones, con excepción de los dispositivos con activación de $9 \mathrm{~mm}$ y espesor de 0,036 pulgadas que mostraron una diferencia estadísticamente significativa $(p<0,05)$ (Tablas I y II). 
Tabla I. Fuerza media (g) producida por los resortes de Coffin. $\mathrm{n}=15$ para cada combinación de aleaciones metálicas y diámetro del alambre utilizado. Letras diferentes indican diferencia estadísticamente significativa $(p<0,05)$ entre los aparato para una misma activación.

\begin{tabular}{lccrrr}
\hline \multicolumn{1}{c}{ Resorte de Coffin } & \multicolumn{4}{c}{ Activación } \\
\hline Liga metálica & $\begin{array}{c}\text { Diámetro } \\
\text { del hilo }\end{array}$ & $\mathbf{4 ~} \mathbf{~ m m}$ & $\mathbf{6 ~} \mathbf{~ m m}$ & $\mathbf{9} \mathbf{~ m m}$ & $\mathbf{1 2} \mathbf{~ m m}$ \\
& 0.032 & $200.2 \pm 22 \mathrm{a}$ & $263.4 \pm 26 \mathrm{a}$ & $361.79 \pm 24 \mathrm{a}$ & $432 \pm 24 \mathrm{a}$ \\
Acero inoxidable & 0.036 & $281 \pm 11 \mathrm{~b}$ & $375.8 \pm 15 \mathrm{~b}$ & $516.3 \pm 28 \mathrm{~b}$ & $653.3 \pm 29 \mathrm{~b}$ \\
Acero inoxidable & 0.032 & $203.7 \pm 0 \mathrm{a}$ & $266.9 \pm 6 \mathrm{a}$ & $358.2 \pm 14 \mathrm{a}$ & $435.5 \pm 16 \mathrm{a}$ \\
Acero inoxidable con bajo contenido de níquel & 0.036 & $298.5 \pm 19 \mathrm{~b}$ & $407.4 \pm 24 \mathrm{~b}$ & $576 \pm 31 \mathrm{c}$ & $730.6 \pm 35 \mathrm{~b}$ \\
\hline Acero inoxidable con bajo contenido de níquel & & &
\end{tabular}

Tabla II. Resistencia y el módulo de elasticidad producida por el resorte de Coffin. $n=15$, para cada combinación entre aleación metálica y diámetro del alambre utilizado. Letras diferentes indican diferencia estadísticamente significativa $(p<0,05)$.

\begin{tabular}{|c|c|c|c|}
\hline \multicolumn{4}{|c|}{ Resorte de Coffin } \\
\hline Liga metálica & $\begin{array}{l}\text { Diámetro del } \\
\text { hilo }\end{array}$ & $\begin{array}{l}\text { Resiliencia } \\
\text { (g.cm) }\end{array}$ & $\begin{array}{c}\text { Módulo de elasticidad } \\
\left(\mathrm{g} / \mathrm{cm}^{2}\right)\end{array}$ \\
\hline Acero inoxidable & 0.032 & $294.8 \pm 17 a$ & $57.14 \pm 9 a$ \\
\hline & 0.036 & $421.6 \pm 22 b$ & $66.65 \pm 12 b$ \\
\hline $\begin{array}{l}\text { Acero inoxidable con bajo contenido } \\
\text { de níquel }\end{array}$ & $\begin{array}{l}0.032 \\
0.036\end{array}$ & $\begin{aligned} 296.3 & \pm 9 a \\
464 & +24 b\end{aligned}$ & $\begin{array}{l}57.43 \pm 19 a \\
73.43+22 b\end{array}$ \\
\hline
\end{tabular}

\section{DISCUSIÓN}

En el presente estudio, los resortes de Coffin mostraron diferentes propiedades mecánicas, lo que indica la necesidad de conocimiento del desempeño de los mismos para cada activación. Además, es importante identificar la etiología de la maloclusión y determinar la fuerza ideal para cada tratamiento (Graber, 1969). El movimiento de un solo molar puede necesitar $250 \mathrm{~g}$ (Jarabak \& Fizzell, 1975), pero se han observado efectos ortopédicos en la dentición temporal y mixta, con fuerzas superiores a $400 \mathrm{~g}$ (Graber, 1985).

Según Proffit el éxito de los aparatos removibles depende de la cooperación del paciente y control de la fuerza del aparato. Para Adams, la cantidad de activación del resorte de Coffin depende del diámetro del alambre, de la longitud del arco y el número de dientes que deban desplazarse. Una activación de 2 a 4 $\mathrm{mm}$ utilizando un alambre de 0,050 pulgadas se ha propuesto como suficiente en el comienzo. Si se necesitan más ajustes, pueden hacerse más tarde. Esta activación está en concordancia con el presente estudio, pero la necesidad de utilizar un alambre de diámetro fino también es sugerido cuando la etiología es una anomalía dentaria. Se observó en este estudio, que las medias de los valores de fuerza aumentaron proporcionalmente a la activación, lo que concuerda con que los aparatos funcionaran en la fase elástica, en la que la deformación es proporcional a la fuerza.
Los resultados mostraron que la mordida cruzada de un solo molar o un grupo pequeño de dientes obtienen un tratamiento adecuado con un resorte de Coffin confeccionado con alambre de 0,032 pulgadas, con 4 a $6 \mathrm{~mm}$ de activación independiente de la aleación metálica utilizada. Cuando un efecto ortopédico pequeño se espera durante la dentición decidua y mixta, el resorte de Coffin confeccionado con alambre de 0,032 pulgadas, con 9 a $12 \mathrm{~mm}$ de activación para las dos aleaciones, de acero inoxidable o de acero inoxidable con bajo contenido de níquel parecen ser la mejor elección. Los resultados sugieren que la activación de 6 a $9 \mathrm{~mm}$ en resortes de Coffin confeccionados con alambre de 0,036 de aleación de acero inoxidable o de aleación de acero inoxidable con bajo contenido de níquel, pueden lograr efectos ortopédicos, cuando se asocia con el uso de equipo fijo.

Los resultados observados en este estudio demostraron que la aleación de alambre de acero inoxidable con bajo contenido de níquel, posee características mecánicas similares al alambre de acero inoxidable convencional y puede ser utilizado en pacientes con antecedentes de hipersensibilidad al níquel, pero se debe estar atento a la activación que se vaya a utilizar, ya que los aparatos con esta aleación mostraron una mayor liberación de fuerza, resistencia y módulo de elasticidad, lo que puede influir en el movimiento dentario. 
El níquel es conocido por su potencial alérgeno (Jacobsen \& Hensten-Pettersen, 1989; Peltonen, 1979; Schubert et al., 1987). Dada la presencia de iones de metales como el níquel en los aparatos de ortodoncia, se ha asociado con reacciones de hipersensibilidad en ortodoncia (Bass et al., 1993). Las manifestaciones clínicas de hipersensibilidad al níquel son fáciles de diagnosticar, frente a un cuadro de alergia cualquier aparato extraoral o intraoral en uso por el paciente que posee níquel debe ser removido hasta que los signos de reacciones adversas en la mucosa o la piel han sanado completamente (Eliades \& Athanasiou).
Una historia previa de alergia debería considerarse un factor predictivo de manifestaciones clínicas de hipersensibilidad al níquel (Genelhu et al., 2005). De esta forma, en lugar de utilizar aparatos intra y extraorales que posee níquel, se sugiere sustituir por braquets y alambre de acero inoxidable libres de níquel de bajo contenido de este ión.

Se puede concluir que los resortes de Coffin con ambos alambres evaluados produjeron fuerzas adecuadas para el tratamiento ortodóntico, a condición de que, sea adecuadamente planificada su aplicación clínica.

DOS SANTOS, R. L. \& PITHON, M. M. Coffin Appliance: Study of 2 different types of metal alloys. Int. J. Odontostomat., 4(2):133-137, 2010.

ABSTRACT: The emergence of stainless steel wire made of low-nickel content was a major breakthrough in the orthodontic, supplying the need for orthodontics patients who may have hypersensitivity by this ion, but do not know much about the mechanical properties of these wires. The objective of this study is to test the hypothesis that there is no difference between stainless steel wires and low-nickel stainless steel ones regarding their mechanical behaviour. Force, resilience, and elasticity modulus produced by Coffin appliances made of 0.032 -inch and 0.036 -inch wires were evaluated. Sixty appliances Coffin were made, thirty for each type of alloy being fifteen for each wire thickness. All the arches were submitted to mechanical compression test by using an EMIC DL-10000 machine simulating activations of 4, 6, 9, and 12 mm. Analysis of variance (ANOVA) with multiple comparisons and Tukey's test were employed $(p<0.05)$ for assessing force, resilience, and elasticity modulus. The groups using the 0.036 inch presented statistically $(p<0.05)$ higher levels of force, resiliency and elasticity modulus when compared to the arches using the 0.032 inch wire for both alloys. The Coffin appliances for both alloys evaluated can produce adequate forces for orthodontic treatment as long as their clinical application is correctly planned.

KEY WORDS: cross-bite, orthodontic appliances, force, nickel allergy.

\section{REFERENCIAS BIBLIOGRÁFICAS}

Adams, C. P. The Design and Construction of Removable Orthodontic Appliances. Bristol, John Wright and Sons, 1969.

Bass, J. K.; Fine, H. \& Cisneros, G. J. Nickel hypersensitivity in the orthodontic patient. Am. J. Orthod. Dentofacial Orthop., 103(3):280-5,1993.

Bell, R. A. \& LeCompte, E. J. The effects of maxillary expansion using a quad-helix appliance during the deciduous and mixed dentitions. Am. J. Orthod., 79(2):152-61,1981.

Chaconas, S. J. \& de Alba y Levy, J. A. Orthopedic and orthodontic applications of the quad-helix appliance. Am. J. Orthod., 72(4):422-8, 1977.

Eliades, T. \& Athanasiou, A. E. In vivo aging of orthodontic alloys: implications for corrosion potential, nickel release, and biocompatibility. Angle Orthod., 72(3):222-37, 2002.

Genelhu, M. C.; Marigo, M.; Alves-Oliveira, L. F.;Malaquias, L. C. \& Gomez, R. S. Characterization of nickel-induced allergic contact stomatitis associated with fixed orthodontic appliances. Am. J. Orthod. Dentofacial Orthop., 128(3):378-81, 2005.

Graber, T. M. Orthodontics. Current Principles and Prattice. St. Louis, Mosby Co, 1985. pp.60-2.

Graber, T. M. Current Orthodontics. Concept and Technique. Philadelphia, WB Saunders Co., 1969. pp.919-88.

Hensten-Pettersen, A.; Jacobsen, N. \& Grímssdóttir, M. R. Allergic reactions and safety concerns: In: 
Brantley, W. A. \& Eliades, T. (Eds). Orthodontic Materials: Scientific and Clinical Aspects. Stuttgart, Thieme, 2001. pp.287-99.

Jacobsen, N. \& Hensten-Pettersen, A. Occupational health problems and adverse patient reactions in orthodontics. Eur. J. Orthod., 11(3):254-64, 1989.

Janson, G. R.; Dainesi, E. A.; Consolaro, A.; Woodside, D. G. \& de Freitas, M. R. Nickel hypersensitivity reaction before, during, and after orthodontic therapy. Am. J. Orthod. Dentofacial Orthop., 113(6):655-60, 1998.

Jarabak, J. R. \& Fizzell, J. A. Aparatología del arco de canto com alambres delgados. Buenos Aires, Mundi, 1975.

Martinelli, F. L.; Couto, P. S. \& Ruellas, A. C. Three palatal arches used to correct posterior dental crossbites. Angle Orthod., 76(6):1047-51, 2006.

Park, H. Y. \& Shearer, T. R. In vitro release of nickel and chromium from simulated orthodontic appliances. Am. J. Orthod., 84(2):156-9, 1983.

Peltonen, L. Nickel sensitivity in the general population. Contact Dermatitis, 5(1):27-32, 1979.

Proffit, W. R. Contemporary Orthodontics. St. Louis, Mosby Co., 1986. pp.341-9.

Schubert, H.; Berova, N.; Czernielewski, A.; Hegyi, E.; Jirasek, L.; Kohanka, V.; Korossy, S.; Michailov, P.; Nebenfuhrer, L. \& Prater, E. Epidemiology of nickel allergy. Contact Dermatitis, 16(3):122-8, 1987.
Dirección para correspondencia:

Rogério Lacerda dos Santos

Praça José Batista de Freitas, 78, Sala 102, Centro, CEP 35519-000

Nova Serrana - MG

BRASIL

\section{Email: lacerdaorto@hotmail.com} lacerdaorto@bol.com.br

Recibido:

Aceptado: 
